\title{
Editorial
}

\section{The Role of Adipose Tissue Lipolysis in Diet-Induced Obesity: Focus on Vimentin}

\author{
Eun Roh, Hye Jin Yoo \\ Division of Endocrinology and Metabolism, Department of Internal Medicine, Korea University College of Medicine, Seoul, Korea
}

Adipose tissue (AT) is the main organ for energy storage. During periods of energy demands, such as fasting and physical exercise, fatty acids (FAs) can be mobilized from fat stores to meet energy needs. Lipolysis is the catabolic pathway through which stored triglycerides (TGs) are hydrolyzed into FA and glycerol. Three lipases work sequentially [1]. First, adipose triglyceride lipase (ATGL) hydrolyzes TG into diacylglycerol (DAG) and FA. Then, hormone-sensitive lipase (HSL) cleaves DAG into monoacylglycerol, which is ultimately converted into FA and glycerol by monoacylglycerol lipase. HSL resides freely in the cytosol and can associate with lipid droplets. Lipolytic hormones such as catecholamines stimulate lipolysis primarily via cyclic adenosine monophosphate (cAMP)-mediated activation of protein kinase A (PKA) [2]. PKA then phosphorylates HSL serine residues 563,659 , and 660 , leading to translocation of HSL to a lipid droplet and to its active participation in lipolysis [3]. On the other hand, AMP-activated protein kinase (AMPK) phosphorylates HSL Ser565 and prevents PKA-mediated phosphorylation of this enzyme [3]. In addition to the cAMP/PKA signaling cascade, pathways involving cGMP-dependent protein kinase, protein kinase $\mathrm{C}$ ( $\mathrm{PKC})$, and extracellular signal-regulated kinase (ERK) contribute to activate lipolysis in adipocytes [4].

During the development of obesity, AT expands tremendously and adipocyte size increases to neutralize and store nutritional overload [5]. This process is necessary to protect the body from peripheral insulin resistance. However, when the adipocytes are eventually unable to store excess lipids, two im- portant pathological processes in AT are important for the development of metabolic diseases: AT inflammation and hypertrophy [5]. In obese individuals, AT inflammation due to the recruitment of T-cells and macrophages has been shown to contribute to insulin resistance by disturbed adipokine balance $[6,7]$. Moreover, large adipocytes increase lipolysis and promote elevated circulating TGs, non-esterified fatty acids (NEFAs) and glycerols. This process is accompanied by redirection of FA to the liver, promoting hepatic TG accumulation [8].

Vimentin is a type III intermediate filament that comprises a type of cytoskeletal element [9]. It is expressed in mesenchymal cells, including adipocytes where it forms lipid droplets, stabilizes TG [10], and participates insulin-dependent translocation of glucose transporter type 4 (GLUT4), the predominant insulin-responsive glucose transporter isoform, to the plasma membrane [11]. Using a proteomics approach, vimentin has been considered to participate in lipolysis through direct interactions with HSL [10], in addition to its interaction with $\beta$-adrenergic receptors and ERK signaling [12]. A recent study showed that mice lacking vimentin $\left(\mathrm{Vim}^{--}\right)$had less fat accumulation compared with wild-type mice, suggesting that vimentin is important for normal fat accumulation in the body [13]. However, there have been no animal studies that verified the role of vimentin in diet-induced obesity and type 2 diabetes mellitus.

In the article titled, "Vimentin deficiency prevents high-fat diet-induced obesity and insulin resistance in mice," Kim et al. [14] investigated whether vimentin deficiency affected high-fat
Corresponding author: Hye Jin Yoo (iD https://orcid.org/0000-0003-0600-0266 Division of Endocrinology and Metabolism, Department of Internal Medicine, Korea University Guro Hospital, Korea University College of Medicine, 148 Gurodong-ro, Gurogu, Seoul 08308, Korea

E-mail: deisy21@naver.com
This is an Open Access article distributed under the terms of the Creative Commons Attribution Non-Commercial License (https://creativecommons.org/licenses/by-nc/4.0/) which permits unrestricted non-commercial use, distribution, and reproduction in any medium, provided the original work is properly cited. 
diet (HFD)-induced obesity and glucose intolerance. After 10 weeks of HFD feeding, $\mathrm{Vim}^{-1-}$ mice showed reduced increase in body weight and less epididymal white AT and subcutaneous AT compared to wild-type mice. Interestingly, epididymal $\mathrm{Vim}^{-/}$adipocytes were bigger than wild-type cells and serum TG and NEFA levels were higher in $\mathrm{Vim}^{-/-}$mice. These results suggested that $\mathrm{Vim}^{-/-}$adipocytes in epididymal fat appear to have improved function with respect to storing lipids and decreasing lipolysis. The authors further revealed that vimentin deficiency reduced trafficking of cluster of differentiation 36 (CD36), a major FA translocase, to the plasma membrane in adipocytes, indicating that vimentin plays a role in trafficking of CD36 and regulates FA uptake of adipocytes. In vimentinnull AT, active forms of HSL phosphorylation (HSL phosphorylation on Ser563, Ser659, and Ser660) were reduced without changes in the expression of FA synthase or perilipin, two major markers for lipogenesis. The authors also evaluated if vimentin affected insulin resistance in HFD-fed obese mice. $\mathrm{Vim}^{-1-}$ mice had significantly lower fasting glucose levels and improved glucose tolerance compared to control mice. For the mechanism, vimentin deficiency resulted in reduced expression of GLUT4 in the plasma membrane in adipocytes, although the role of vimentin in different regulatory mechanisms should be studied.

CD36, a multifunctional membrane receptor, is a key facilitator of FA uptake and subsequent TG storage in adipocytes [15]. CD36 localization at the plasma membrane was shown to be critical for its activity in FA uptake [16]. CD36 deletion in 3T3-L1 adipocytes decreased lipolysis with altered signaling in the cAMP/PKA and ERK pathways [16]. In humans with obesity and type 2 diabetes mellitus, subcutaneous AT CD36 expression was upregulated, whereas visceral AT CD36 expression was only increased in type 2 diabetes mellitus [17]. CD36 deficient mice showed reduced adiposity and improved insulin resistance [18]. Kim et al. [14] showed that vimentin-null adipocytes have less membrane-localized CD36 and thus less FA uptake via CD36. This deficient CD36-mediated signaling may contribute to decreased lipolysis via reduced phosphorylation of HSL. Thus, we can conclude that vimentin should be noted as a new therapeutic target for diet-induced obesity and diabetes through the regulation of CD36 trafficking and FA uptake in adipocytes. Further studies are needed to elucidate the mechanism by which vimentin is involved in membrane localization of CD36.

\section{CONFLICTS OF INTEREST}

No potential conflict of interest relevant to this article was reported.

\section{REFERENCES}

1. Nielsen TS, Jessen N, Jorgensen JO, Moller N, Lund S. Dissecting adipose tissue lipolysis: molecular regulation and implications for metabolic disease. J Mol Endocrinol 2014;52:R199222.

2. Arner P. Catecholamine-induced lipolysis in obesity. Int J Obes Relat Metab Disord 1999;23 Suppl 1:10-3.

3. Gaidhu MP, Anthony NM, Patel P, Hawke TJ, Ceddia RB. Dysregulation of lipolysis and lipid metabolism in visceral and subcutaneous adipocytes by high-fat diet: role of ATGL, HSL, and AMPK. Am J Physiol Cell Physiol 2010;298:C961-71.

4. Carmen GY, Victor SM. Signalling mechanisms regulating lipolysis. Cell Signal 2006;18:401-8.

5. Sun K, Kusminski CM, Scherer PE. Adipose tissue remodeling and obesity. J Clin Invest 2011;121:2094-101.

6. Trayhurn P, Wood IS. Adipokines: inflammation and the pleiotropic role of white adipose tissue. Br J Nutr 2004;92:347-55.

7. Strissel KJ, Stancheva Z, Miyoshi H, Perfield JW 2nd, DeFuria J, Jick Z, et al. Adipocyte death, adipose tissue remodeling, and obesity complications. Diabetes 2007;56:2910-8.

8. Fabbrini E, Sullivan S, Klein S. Obesity and nonalcoholic fatty liver disease: biochemical, metabolic, and clinical implications. Hepatology 2010;51:679-89.

9. Fuchs E, Weber K. Intermediate filaments: structure, dynamics, function, and disease. Annu Rev Biochem 1994;63:345-82.

10. Shen WJ, Patel S, Eriksson JE, Kraemer FB. Vimentin is a functional partner of hormone sensitive lipase and facilitates lipolysis. J Proteome Res 2010;9:1786-94.

11. Hirata Y, Hosaka T, Iwata T, Le CT, Jambaldorj B, Teshigawara $\mathrm{K}$, et al. Vimentin binds IRAP and is involved in GLUT4 vesicle trafficking. Biochem Biophys Res Commun 2011;405:96101.

12. Kumar N, Robidoux J, Daniel KW, Guzman G, Floering LM, Collins S. Requirement of vimentin filament assembly for beta3-adrenergic receptor activation of ERK MAP kinase and lipolysis. J Biol Chem 2007;282:9244-50.

13. Wilhelmsson U, Stillemark-Billton P, Boren J, Pekny M. Vimentin is required for normal accumulation of body fat. Biol Chem 2019;400:1157-62. 
14. Kim S, Kim I, Cho W, Oh GT, Park YM. Vimentin deficiency prevents high-fat diet-induced obesity and insulin resistance in mice. Diabetes Metab J 2021;45:97-108.

15. Su X, Abumrad NA. Cellular fatty acid uptake: a pathway under construction. Trends Endocrinol Metab 2009;20:72-7.

16. Zhou D, Samovski D, Okunade AL, Stahl PD, Abumrad NA, $\mathrm{Su}$ X. CD36 level and trafficking are determinants of lipolysis in adipocytes. FASEB J 2012;26:4733-42.
17. Bonen A, Tandon NN, Glatz JF, Luiken JJ, Heigenhauser GJ. The fatty acid transporter FAT/CD36 is upregulated in subcutaneous and visceral adipose tissues in human obesity and type 2 diabetes. Int J Obes (Lond) 2006;30:877-83.

18. Hajri T, Han XX, Bonen A, Abumrad NA. Defective fatty acid uptake modulates insulin responsiveness and metabolic responses to diet in CD36-null mice. J Clin Invest 2002;109: 1381-9. 